\title{
Vertical Launch Third Harmonic Electron Cyclotron Resonance Heating of H-mode on TCV and Access to Quasi-Stationary
}

\section{ELM-free H-mode}

L. Porte, S. Alberti, E. Asp, G. Arnoux (a) ${ }^{(a)}$ A. Bortolon, Y. Martin, M. Maslov, O. Sauter, A. Scarabosio, H. Weisen

Ecole Polytechnique Fédérale de Lausanne (EPFL), Centre de Recherches en Physique des Plasmas, Association EURATOM - Confédération Suisse, 1015 Lausanne, Switzerland

(a) Départment de Recherches sur la Fusion Contrôlée, Association EURATOM-CEA, CEA/Cadarache, 13108 Saint Paul-lez-Durance Cédex, France

The Tokamak à Configuration Variable (TCV) is equipped with three 480kW gyrotrons operating at 118GHz. Radiation is launched in the extraordinary mode allowing plasma heating using third harmonic X-mode electron cyclotron resonance heating (ECRH) at density above X2 cut-off. This system (X3) [1] allows high beta and ion heating, through electron-ion collisions, to be produced. Power is transmitted to the tokamak along 3 evacuated waveguides where it is projected onto one plasma facing mirror that can be translated radially, between shots, and rotated poloidally during a shot.

Linear ray-tracing [2] has been shown [3] to be an adequate tool for estimating the X3 coupled power in H-mode plasma and all estimates of X3 coupled power presented in this paper have been obtained using ray tracing.

Experiments were performed to heat H-mode using X3. The target was an ohmic H-mode. Up to 90\% of the launched X3 power was coupled to the plasma so that the total heating power (1.5MW) was about three times greater than the ohmic H-mode threshold power (500kW). This level of coupled power was maintained even in the presence of significant perturbations to the plasma; ELMS. The radiated power was 300kW during the X3 heated phase. Typically, the X3 heated H-mode discharges entered a large ELMy regime where the energy loss per ELM was $\approx 12 \%$. The electron temperature increased from $1 \mathrm{keV}$ to $3 \mathrm{keV}$ and the stored energy and $\beta_{\mathrm{N}}$ were doubled. The energy confinement time in the large ELM phase was up to 25msec $\left(\mathrm{H}_{\mathrm{IPB}(\mathrm{y}, 2)}=1.3\right)$ and the high confinement was maintained for 30 confinement times. On other occasions the X3 heated $\mathrm{H}$ modes transited into an ELM free H-mode regime with constant electron density and stored energy. During the X3 phase the stored energy and $\beta_{\text {tor }}$ both doubled $\left(\beta_{\mathrm{N}}=2\right)$. The maximum, achieved $\beta_{\text {tor }}$ was $2.5 \%$ while the ideal $\beta$-limit for these discharges was $3.5 \%$. The recycling light level was high compared to the baseline ohmic H-mode level and the fluctuations in the recycling light level were correlated with core MHD. The confinement time for these discharges was found to be as high as $30 \mathrm{~ms}\left(\mathrm{H}_{\mathrm{IPB}(\mathrm{y}, 2)}=1.7\right)$. Measurements of carbon ion temperature profiles and carbon rotation velocity using charge exchange recombination spectroscopy were made and during the quiescent phase the ion temperature, near mid radius, increased from $500 \mathrm{eV}$ to $1 \mathrm{keV}$. The plasma rotation increased also from $5 \mathrm{kms}^{-1}$ to $50 \mathrm{kms}^{-1}$ in the direction of plasma current.

[1] S. ALBERTI et al, Nucl. Fusion 45, 1224 (2005)

[2] K. MATSUDA, IEEE Trans. Plasma Sci. 176 (1989)

[3] G. ARNOUX Ph.D. Thesis 3401, Ecole Polytechnique Fédérale de Lausanne (2005). 